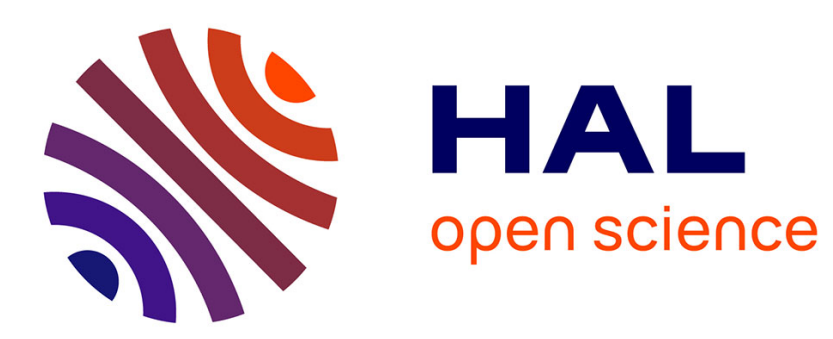

\title{
Sexualité juvénile sous contrôle dans les écoles secondaires maliennes (1960-1970)
}

Ophélie Rillon

\section{To cite this version:}

Ophélie Rillon. Sexualité juvénile sous contrôle dans les écoles secondaires maliennes (1960-1970).

Clio. Femmes, Genre, Histoire, 2015, Âge et sexualité, 42, pp.77-97. 10.4000/clio.12757 . hal01539900

\section{HAL Id: hal-01539900 \\ https://hal.science/hal-01539900}

Submitted on 15 Jun 2017

HAL is a multi-disciplinary open access archive for the deposit and dissemination of scientific research documents, whether they are published or not. The documents may come from teaching and research institutions in France or abroad, or from public or private research centers.
L'archive ouverte pluridisciplinaire HAL, est destinée au dépôt et à la diffusion de documents scientifiques de niveau recherche, publiés ou non, émanant des établissements d'enseignement et de recherche français ou étrangers, des laboratoires publics ou privés. 


\title{
Clio. Femmes, Genre, Histoire
}

$42(2015)$

Âge et sexualité

Ophélie Rillon

\section{Sexualité juvénile sous contrôle dans les écoles secondaires maliennes (1960-1970)}

\author{
Avertissement \\ Le contenu de ce site relève de la législation française sur la propriété intellectuelle et est la propriété exclusive de \\ l'éditeur. \\ Les œuvres figurant sur ce site peuvent être consultées et reproduites sur un support papier ou numérique sous \\ réserve qu'elles soient strictement réservées à un usage soit personnel, soit scientifique ou pédagogique excluant \\ toute exploitation commerciale. La reproduction devra obligatoirement mentionner l'éditeur, le nom de la revue, \\ l'auteur et la référence du document. \\ Toute autre reproduction est interdite sauf accord préalable de l'éditeur, en dehors des cas prévus par la législation \\ en vigueur en France.
}

\section{revues.org}

Revues.org est un portail de revues en sciences humaines et sociales développé par le Cléo, Centre pour l'édition électronique ouverte (CNRS, EHESS, UP, UAPV).

\section{Référence électronique}

Ophélie Rillon, « Sexualité juvénile sous contrôle dans les écoles secondaires maliennes (1960-1970) », Clio.

Femmes, Genre, Histoire [En ligne], 42 | 2015, mis en ligne le 01 décembre 2018, consulté le 10 mars 2016. URL:

http://clio.revues.org/12757; DOI : 10.4000/clio.12757

Éditeur : Éditions Belin
http://clio.revues.org
http://www.revues.org

Document accessible en ligne sur : http://clio.revues.org/12757

Ce document est le fac-similé de l'édition papier.

Cet article a été téléchargé sur le portail Cairn (http://www.cairn.info).

\section{CAIRN}

Chercher. Repérer. Avancer.

Distribution électronique Cairn pour Éditions Belin et pour Revues.org (Centre pour l'édition électronique ouverte) Tous droits réservés 


\section{Sexualité juvénile sous contrôle dans les écoles secondaires maliennes (1960-1970)}

Ophélie RILLON ${ }^{1}$

«Les filles écolières deviennent quasi-totalité prostituées $[$ sic $] »^{2}$. Cette allégation, formulée par un journal musulman soudanais en 1957, témoigne des violentes réactions suscitées par la scolarisation des filles en Afrique occidentale française (AOF). L'assimilation de l'école à un lieu de débauche sexuelle pour les jeunes filles n'était certes pas un phénomène nouveau à la fin des années 1950. Dès l'origine, de telles rumeurs ont accompagné la politique scolaire initiée par l'administration coloniale dans l'entre-deux-guerres. La romancière sénégalaise Mariama Ba se souvient ainsi que, dans les années 1940, l'école était accusée de transformer « les filles en diablesses qui détournent les hommes du droit chemin $»^{3}$; et l'historienne Pascale Barthélémy rapporte le conseil formulé par une mère dahoméenne à sa fille en partance pour l'école normale de Rufisque (Sénégal): "Amélie il faut toujours porter ta culotte hein !! $»^{4}$. Le problème ainsi soulevé par les adultes était que l'entrée à l'école repoussait l'âge au mariage des filles et les détachait du contrôle parental pour les placer en internat, loin du domicile familial. Cette infime minorité de filles scolarisées bénéficiait d'une période de célibat qui se prolongeait bien au-delà de 14 ans $^{5}$ et leur ouvrait la

1 Je remercie particulièrement Anne Hugon pour ses commentaires et précieux conseils qui ont accompagné la rédaction de cet article.

2 Presse divine, $\mathrm{n}^{\circ} 53,1^{\text {er }}$ août 1957, p. 2.

3 Bâ $2007: 40$.

4 Barthélémy 2004 : 410.

5 Âge légal du mariage pour les filles instauré par le décret Mandel de 1937. Ce décret était en réalité peu appliqué et nombre de jeunes filles non scolarisées étaient mariées plus jeunes. 
possibilité d'une vie sexuelle (en dépit de la non-mixité de l'internat) sans encadrement conjugal. Si certaines d'entre elles expérimentaient premières amours et initiation sexuelle durant leur formation - comme en témoignent les affaires d'exclusion d'élèves enceintes des écoles fédérales étudiées par P. Barthélémy - rien n’indique pourtant que la scolarisation des filles allait de pair avec un rajeunissement de leur entrée en sexualité, bien au contraire ${ }^{6}$. Une riche historiographie a ainsi montré comment, dans les différents empires coloniaux, l'administration avait contribué à forger, de concert avec les chefs locaux, la représentation d'une déviance féminine centrée sur la sexualité où étaient stigmatisés pêle-mêle la prostitution, l'adultère et le célibat féminin ${ }^{7}$, en somme tout ce qui marquait un éloignement au mariage.

Dans l'ancien Soudan français (actuel Mali), les craintes suscitées par l'autonomie sexuelle des écolières ont connu un nouvel essor dans le contexte de la lutte anticoloniale et avec l'arrivée au pouvoir du Rassemblement démocratique africain (RDA) en 1956. Aux yeux des dirigeants africains, filles et garçons de la jeune génération constituaient le terreau dont devait surgir la nouvelle société malienne. L'école et les organisations de jeunesses socialistes, qui virent le jour au tournant de l'indépendance en 1960, étaient conçues comme les instruments de formation des forces vives de la nation, dont la vigueur physique et la bonne moralité ne pouvaient être suspectées. Ainsi, en même temps qu'émergeait en milieu urbain "une culture juvénile adolescente ${ }^{8}$ favorisée par la sociabilité scolaire et celle des organisations de jeunesse, l'idéal d'une jeunesse disciplinée et aux ordres du Parti accentua l'encadrement des comportements juvéniles et la traque de leurs relations charnelles. Dans les années 1960-1970, la question de la sexualité des élèves - et des filles en particulier - a largement débordé le cadre des institutions

6 Les enquêtes démographiques et de santé (EDS) menées au Mali depuis les années 1990 montrent, à l'inverse, que les femmes diplômées du secondaire débutent en moyenne leur vie sexuelle à 18 ans, soit 2,5 ans plus tard que les femmes non scolarisées. En outre leur entrée en union s'effectuait à 20,5 ans, soit cinq années plus tard que les femmes non scolarisées. République du Mali 1996 ; République du Mali 2006.

7 Allman 1996 ; Thomas 2006a ; Goerg 2007 ; Jean-Baptiste 2014.

8 Morin 1969 ; Sohn 2001. 
scolaires pour devenir la cible d'une mobilisation sociale et morale. L'importance accordée à ce sujet dans le champ médiatique, politique et sanitaire allait de pair avec la construction d'une menace juvénile. Comme dans d'autres régions du monde à la même période, les jeunes Africains issus des milieux urbains et scolaires se sont progressivement imposés comme des acteurs sociaux autonomes à partir des années $1945^{9}$, générant des peurs profondément genrées. Si la capacité de violence politique et collective des jeunes hommes était crainte par les autorités coloniales puis nationales, c'était l'autonomie sexuelle d'individus-filles qui préoccupaient les esprits des aîné-e-s.

En croisant l'analyse des archives administratives (policières, scolaires, sanitaires, ou archives du parti unique), celles du journal gouvernemental (seul organe de presse autorisé à partir de 1960) et des sources orales recueillies auprès d'ancien-ne-s lycéen-ne-s et du personnel du planning familial, créé à Bamako au début des années 1970, cet article montre que la sexualité juvénile constitua un enjeu politique crucial tout au long de ces deux décennies, auquel des réponses diverses furent apportées en fonction des régimes. Après avoir étudié comment une revendication d'autonomie sexuelle se fit de plus en plus audible au tournant de l'indépendance dans les établissements scolaires des deux sexes, on examinera en quoi cette question généra une " panique morale ${ }^{10}$ des autorités maliennes. Dans le contexte de transformation révolutionnaire auquel aspiraient les dirigeants de la $1^{\text {re }}$ République socialiste, la quête masculine du plaisir était perçue comme le signe d'une décolonisation inaboutie et de l'échec de leur politique éducative. Le coup d'État militaire de 1968 provoqua un changement radical dans la politique d'encadrement de la sexualité juvénile. À la répression, succéda une gestion sanitaire qui visait à prévenir les grossesses hors-mariages et faisait endosser aux filles l'entière responsabilité des conséquences de l'activité sexuelle.

\footnotetext{
9 Diouf 2003.

10 Bozon 2012.
} 


\section{«On était quand même des jeunes filles... on avait nos petits copains! ». Sociabilités amoureuses au tournant des indépendances}

Le «tout petit monde $»^{11}$ de l'internat qu'avait connu la génération des écoles fédérales avait fortement évolué à la veille des indépendances. Ce n'était plus au Sénégal que les élèves étaient envoyé-e-s pour poursuivre leurs études, mais à Bamako où deux établissements secondaires de filles ont vu le jour dans les années 195012. Si l'élite du lycée de Jeunes Filles vivait en internat avec des autorisations de sorties le week-end, la majeure partie des filles scolarisées habitait chez leurs parents ou dans des familles d'accueil où se côtoyait une jeunesse composite. Ce rapprochement géographique a profondément ouvert la marge d'autonomie des scolaires qui, du Fondamental au Supérieur ${ }^{13}$, ne formaient plus un groupe social marginal vivant dans un univers clos. La socialisation scolaire, conjuguée à celle des organisations de jeunesse, a favorisé l'émergence d'espaces de rencontres mixtes (socialement et sexuellement) et le développement de loisirs propres aux jeunes. Activités théâtrales et sportives, soirées dansantes, baignades au fleuve Niger et sorties au cinéma étaient autant d'occasions pour les

11 Expression employée par Barthélémy 2004 : 388.

12 Le collège moderne des jeunes filles fut créé en 1951 et transformé en lycée en 1959. Les classes ouvertes par les missions catholiques donnèrent naissance au lycée Notre Dame du Niger pour les filles (officiellement inauguré en 1959) et au lycée Prosper Camara pour les garçons (officiellement inauguré en 1963). La création de ces trois établissements secondaires vint ainsi accroître les effectifs scolaires à Bamako où n'existait, depuis 1923, que l'école de formation des cadres masculins : le lycée Terrasson de Fougères, renommé lycée Askia Mohammed en 1961.

13 L'entrée des enfants au cycle fondamental s'étalait entre 6 et 10 ans en fonction des places disponibles et de l'appui familial à la scolarisation. Ce phénomène, conjugué à la déperdition scolaire et aux multiples redoublements, pouvait conduire les jeunes, et les filles en particulier, à fréquenter le cycle fondamental (équivalent primaire et collège) à l'âge où leurs camarades, issus notamment des milieux aisés et lettrés, entraient au lycée (16 ans). La socialisation juvénile au Mali transcendait donc largement les niveaux scolaires. Les élèves du Fondamental, du Secondaire voir du Supérieur pouvaient fréquenter les mêmes soirées. 
garçons et les filles de découvrir l'amour et la sexualité ${ }^{14}$. Avoir son "petit copain» s'était progressivement imposé comme un fait largement partagé par les lycéennes. Les débats qui traversaient, à la fin des années 1950, l'association des jeunes filles du lycée catholique «Rencontres Africaines », étaient le reflet d’une libération de la parole féminine sur la sexualité, ainsi que des tensions générées entre le poids de la morale sexuelle et leur aspiration au plaisir ${ }^{15}$ :

Pour nous jeunes filles africaines, nos coutumes et notre simple bon sens défendent tout essai avant le mariage, et nous exigeons qu'en cela notre virginité soit parfaitement respectée [...] Notre attitude de jeunes filles «à marier» et non à «flirt» ou à «essai » s'explique par une réserve nécessaire. Sommes-nous pour cela bêcheuses «ou indifférentes»? Ne pensez-vous pas que ce soit seulement une méfiance bien fondée ? [...] Et là, je ne puis m'abstenir de penser à l'une de vos expressions favorites $[\ldots]$ «Les filles c'est juste bon pour danser $»^{16}$.

C'est par ces mots qu'une étudiante de passage à Bamako s'adressait aux élèves des établissements secondaires, à l'occasion d'une conférence organisée par les lycéennes en 1957. Indirectement, cette étudiante mettait en exergue l'asymétrie de genre face aux premières expériences sexuelles, sans ouvertement la critiquer. Elle enjoignait ses cadettes à la retenue et interpellait leurs camarades masculins pour leur demander de respecter ce choix de virginité. Un choix, rappelait-elle, qui résultait moins d'une velléité féminine que des conventions sociales qui entouraient leur sexualité. Le sérieux dont devaient faire preuve les jeunes filles était envisagé dans l'ensemble de leurs relations avec les garçons, et le glissement effectué entre la sexualité et la danse témoigne de l'importance de ce loisir dans l'initiation graduelle au désir. Un an plus tard cependant, c'est sur un tout autre registre que le journal des jeunes filles du lycée catholique sermonnait ses lectrices, à la suite d'une soirée où les lycéennes s'étaient fait remarquer pour leur timidité à l'égard des garçons :

14 Rillon 2010.

15 Un phénomène similaire à ce qui se passe en France à la même période : Rebreyend 2010 ; Revenin 2015.

16 «Un problème brûlant traité au Centre d'études et de rencontres africaines : le rôle de la femme africaine dans l'Afrique de demain ", L'Étudiant Soudanais, 1, mai-juin 1957, p. 11-12. 
SOUDANAISES !! écoutez ce que les Sénégalais vous reprochent: ne s'ouvrent pas... un peu distantes... ne discutent pas... refusent même de danser ? EST CE VRAI ? Voir p. 20 le moyen d'y remédier ${ }^{17}$.

La réserve prônée par les aînées avait ainsi cédé le pas à l'injonction au flirt, même si - en apparence du moins - les relations se devaient de rester « chastes ».

Les correspondances entretenues entre le ministère de l'Éducation nationale et le lycée de Jeunes Filles de Bamako montrent à quel point l'encadrement de la sexualité juvénile a pu inquiéter les éducateurs et les responsables politiques, au lendemain de l'indépendance du Mali. Les filles internes ayant passé la nuit en dehors de l'établissement comparaissaient devant un Conseil de discipline et les élèves dont l'état de grossesse était découvert étaient automatiquement exclues ${ }^{18}$. Sira Diop, alors directrice du lycée des filles, n'avait rien trouvé de mieux, pour contrôler la bonne moralité de ses élèves, que d'engager « une équipe de douze [jeunes] hommes du service civique qui veillait bénévolement sur l'établissement de six heures du soir à six heures du matin ${ }^{19}$. Une ancienne lycéenne se souvient ainsi avec indignation des méthodes de contrôle des relations amoureuses et sexuelles instaurées dans les années 1960 :

On était violées dans nos libertés ! Elle [Sira Diop] lisait le courrier ... Ah on n'aimait pas ça $[\ldots]$ On était quand même des jeunes filles ... on faisait le bac, on avait déjà 17-18 ans, on avait nos petits copains! [...] Et puis il y a une autre chose plus grave qu'elle faisait : elle faisait contrôler les règles des filles [...] Il y a des visites médicales qui s'organisent au début de chaque année. Bon il y a des filles qui bien sûr échappent à la vigilance des médecins, qui tombent enceintes et une fois il y a même eu le cas d'un infanticide parce qu'il y a une fille qui a accouché, on a mis l'enfant dans un sac, on l'a mis derrière le mur... Bon tout ça bien sûr a créé une très grande émotion donc tout de suite la réaction a été de mettre un cahier.

17 Rencontres africaines, 1958-septembre, $\mathrm{n}^{\circ} 7$ (à la suite du $1^{\text {er }}$ festival de la jeunesse d'Afrique qui se tint à Bamako du 6 au 11 septembre 1958).

18 Dans les correspondances, ces grossesses étaient pudiquement nommées « raison de santé » et « inaptitudes physiques ». Archives nationales du Mali (ANM), fonds 1920-1960, tome III-B.655, correspondances diverses du lycée de Filles de Bamako 1962-1963.

19 ANM, fonds 1920-1960, tome III-B.655, correspondances diverses du lycée de Filles de Bamako 1962-1963. 
Quand vous avez vos règles, vous allez marquer la date de vos règles ... Ah ça c'était un peu trop fort ... même pour le principe !20.

Si, à l'instar de leurs aînées, les lycéennes des années 1960 n’ont pas mené de luttes collectives pour combattre ces règlements, elles surent user des opportunités que leur offrait l'éducation socialiste pour s'octroyer une marge de liberté accrue. Madina Ly, qui prit la relève de Sira Diop en 1966, évoque ainsi ses difficultés à maintenir l'ordre et le couvre-feu chez des jeunes filles éduquées au marxismeléninisme ${ }^{21}$. Celles-ci s'étaient réapproprié la règle de l'autodiscipline, et en avaient fait une arme pour subvertir le contrôle des adultes et sortir à leur guise. Les garçons du lycée Askia Mohamed, qui disposaient d'une plus grande liberté, n'hésitaient pas, à l'inverse, à se mettre en grève, à envoyer des lettres au gouvernement et à forcer les portes de leur établissement pour manifester leur colère : «Il y a révolution au lycée, car le proviseur a dit qu'on ne sortira pas ce jeudi soir [...] Nous on a défoncé la porte et nous sommes sortis» racontait l'un d'eux dans une lettre à son oncle ${ }^{22}$. Cette révolte lycéenne fut déclenchée en 1962 par une tentative de restriction de leurs sorties les jeudis et samedis soirs, qui les empêchait de voir leurs petites amies et de fréquenter les surprises-parties.

Aujourd'hui, une part de celles et ceux qui étaient adolescents dans les années 1960 jettent un voile pudique sur leurs premières expériences sexuelles. La respectabilité venant avec l'âge, il ne sied pas à des hommes et des femmes marié.e.s, ayant des enfants, voire des petits-enfants, d'évoquer leurs liaisons préconjugales. Pourtant le journal intime d'un lycéen, conservé aux archives nationales maliennes, permet d'appréhender la vie sexuelle et surtout les projections d'un garçon de cette génération ${ }^{23}$. Dans les pages rédigées entre 1963 et 1967, date de la saisie du journal par les services de police, le jeune

20 Élève au lycée de Jeunes Filles de Bamako de 1963 à 1965, Paris, 28 janvier 2013.

21 Entretiens avec Madina Ly, Bamako, septembre 2014.

22 ANM, fonds 1920-1960, tome III, B.1047, correspondances diverses du lycée Askia Mohamed 1961-1963, dossier 1962-novembre, Bamako, 8 novembre 1962, 2 p.

23 Cette archive m'a été signalée par Marie Rodet, que je remercie vivement. ANM, fonds de l'US-RDA BPN, 136e carton : 527, affaire Idrissa Sidibé dit Lambert et Charles Blonda Troaré. 
homme relate ses relations amoureuses en faisant, pour chacune d'entre elles, le compte rendu du "premier rendez-vous», auquel succédait le « premier baiser d'amour » puis la « première nuit d'amour » sans qu'il ne dise rien de ses pratiques sexuelles. Tout en étant une thématique récurrente du journal, la sexualité était toujours articulée au sentiment amoureux et, s'il multipliait les relations, c'était dans l'idéal du couple conjugal que se projetait le lycéen :

Elle m'avait ensorcelé, je ne pouvais plus me séparer d'elle [...] elle était comme ma femme, je couchais toutes les nuits chez elle. Nous partions au ciné, comme 2 jeunes mariés et on passait la nuit dehors, soit au grand hôtel, soit à la carrière qui est à côté de notre école ${ }^{24}$.

La mise en scène de la séduction, des émotions intenses et enfin d'une violence virile à l'encontre de ses partenaires qu'il giflait à la fin de chacune de ses histoires - un acte qui provoquait chez le jeune homme autant de fierté que de remords - convoquait les stéréotypes conjugaux diffusés par les romans-feuilletons français lus par la jeunesse scolaire malienne (Nous Deux, Elle, Intimité, Salut les Copains). Sous sa plume cependant, les jeunes filles n'étaient pas des conquêtes passives: certaines prenaient les devants, le draguaient, lui offraient des cadeaux (vinyles et vêtements à la mode), l'invitaient à passer la nuit dans leur domicile parental ou lui préféraient un autre camarade de classe. Moins nombreuses, les filles scolarisées disposaient d'un «marché matrimonial » beaucoup plus ouvert que les garçons qui se faisaient une rude concurrence pour les séduire. Cette asymétrie atténuait - en partie - l'inégalité des rapports de genre dans l'apprentissage amoureux. L'initiation du jeune homme se révélait ainsi complexe, empreinte de doutes, de passions, de questionnements et de regrets.

\section{Panique morale des adultes}

\section{face à la sexualité juvénile masculine (1967-1968)}

La découverte de ce journal intime, à la fois conforme à l'idéal sentimental hérité des écoles coloniales et qui exposait la banalisation des relations sexuelles juvéniles, provoqua une véritable panique chez

24 ANM, fonds de l'US-RDA BPN, 136e carton : 527, affaire Idrissa Sidibé dit Lambert et Charles Blonda Troaré. 
les autorités maliennes. Celles-ci y virent la preuve d'une "dégénérescence morale» de la jeunesse scolarisée, jusqu'alors considérée comme «l'avant-garde» de la construction nationale. Le contexte politique dans lequel le journal fut rendu public participa largement à en faire une affaire d'État. Il fut, en effet, saisi en 1967 à l'occasion de l'arrestation à la frontière du Sénégal d'un couple de scolaires (une lycéenne mineure, fille de Ministre, et un élève du Cycle Fondamental majeur) qui tentait de fuir le Mali pour poursuivre leurs études en France ${ }^{25}$. Que des jeunes gens appartenant à l'élite nationale cherchent à s'exiler dans l'ancienne puissance coloniale constituait un véritable affront à l'égard du régime socialiste ${ }^{26}$. Si la lycéenne échappa aux poursuites judiciaires grâce à l'intervention de son père, le jeune homme fut inculpé pour "détournement de mineure" avec son ami (auteur du journal) qui les avait aidés à préparer leur expatriation. Cette affaire intervenait dans une période de radicalisation du régime malien après qu'un coup d'État militaire ait renversé le président ghanéen Kwame Nkrumah en 1966. L'événement venait confirmer aux yeux des dirigeants socialistes que les régimes «révolutionnaires» africains étaient menacés de l'intérieur comme de l'extérieur par «des forces rétrogrades ». Prenant modèle sur la révolution culturelle chinoise, ils lancèrent en août 1967 une «révolution active» visant à purger la société de ses «mauvaises graines». S’il ne faudrait pas surestimer l'impact de la saisie du journal sur le basculement du Mali dans cette période de violence politique, la découverte de ce document a certainement accru le poids accordé à la moralisation de la sexualité juvénile au cours de cet épisode révolutionnaire.

Les notes personnelles du lycéen servirent de support à la rédaction d'un Rapport de synthèse sur le problème de l'amoralité et de la licence chez la jeunesse, dans lequel «le désordre des relations sexuelles» s'imposait comme la cause principale du dérèglement moral de la jeunesse ${ }^{27}$. Si

25 ANM, fonds de l'US-RDA BPN, 136 carton : 527, affaire Idrissa Sidibé dit Lambert et Charles Blonda Troaré, extraits de l'enquête préliminaire envoyés par le Président Modibo Keita aux responsables de différents services étatiques, 6 mai 1967,5 p.

26 Rodet \& County 2016 (à paraître); Gary-Tounkara 2003.

27 ANM, fonds de l'US-RDA BPN, 110e carton : 420, rapport de synthèse sur le problème de la moralité et de la licence chez la jeunesse, 1967, 14 p. La 
l'attrait des hommes maliens pour «la femme et les plaisirs afférents $[s i c]$ » était décrit comme "atavique », les autorités se donnaient pour tâche d'en limiter les excès (partenaires multiples, violence). L'enjeu était de contenir ces «manifestations » viriles dans le «cadre légal» du mariage ${ }^{28}$. À lire le rapport, il ressort que la principale préoccupation des autorités politiques était de remettre sur le droit chemin les jeunes hommes et, pour ce faire, d'éliminer les sources du « mal » qui les avait gagnés: les influences occidentales (musique, mode, cinéma), les « lectures pornographiques » (les œuvres de Camus, d'Alfred de Musset et de Baudelaire étaient citées en exemple) et les jeunes filles qui savent « jouer la force [sic] de leurs attraits physiques et vestimentaires ». Avec ce rapport, s'opérait un véritable renversement des représentations. Pour la première fois, c'était la sexualité des garçons qui générait les plus vives inquiétudes: pour que triomphe «l'édification du socialisme ", il fallait les rééduquer, régénérer une masculinité déviante. Dans cette quête de transformation sociale et morale, les jeunes filles n'étaient, certes, pas oubliées, mais c'était à la famille qu'incombait désormais leur éducation. Parents et dirigeants politiques se renvoyaient ainsi la responsabilité de l'encadrement de la sexualité des jeunes filles. Pour les premiers, les mouvements de jeunesse et l'école étaient «créé[s] pour la perversion de nos filles » ${ }^{29}$. Face à la multiplication prétendue des grossesses précoces d'écolières, certains notables engagèrent des actions en justice à l'encontre des enseignants, sans qu'aucune condamnation n'aboutisse ${ }^{30}$. En effet, l'État n'était pas prêt à reconnaître les défaillances de ses organisations de jeunesse ni les

production de ce rapport mobilisa les plus hautes instances de l'État: les commissions (politique, sociale et culturelle, administrative et judiciaire) du Bureau politique, les ministères (Justice, Éducation nationale, Santé publique et Affaires sociales), la Commission nationale de la jeunesse ainsi que les Comités politiques des grandes écoles nationales.

28 ANM, fonds de l'US-RDA BPN, 110e carton : 420, rapport de synthèse sur le problème de la moralité et de la licence chez la jeunesse, 1967, p. 2.

29 Propos tenus par des parents et rapportés par le journal gouvernemental « La soirée des Pionniers ", L'Essor, 17 décembre 1964, p. 3-4.

30 Ministère de l'administration territoriale et des collectivités locales (MATCL), carton N3/20: Renseignements 1962-1969, dossier 343, Revue mensuelle des évènements 1967-1969, Cercle de Kangaba, octobre 1967. 
viols dont des cadres masculins pouvaient se rendre coupables. Aux yeux des dirigeants, le faible écart d'âge entre les enseignants du cycle fondamental et leurs élèves était propice au développement de liaisons, certes immorales, mais dont la faute incombait autant aux écolières. L'enjeu était donc de restaurer l'autorité paternelle afin d'endiguer les pratiques « dégradantes » de ces jeunes filles qui portaient « préjudice au prestige et à la bonne réputation » des institutions socialistes (l'école, les organisations de jeunesse et la milice notamment) ${ }^{31}$.

Pour mener à bien la reconversion morale juvénile, l'organe de presse gouvernemental appela les jeunes à constituer des Brigades de mœurs pour traquer, dans les lieux publics comme privés, les couples adultères et les jeunes aux comportements déviants (mini-jupes, coupes afros et pantalons patte d'éléphant en étaient le symbole) ${ }^{32}$. L'action «musclée» des jeunes militants révolutionnaires s'accompagna de violences à caractère sexuel, notamment à l'égard des filles arrêtées : vêtements déchirés, insultes, menaces et parfois viols $^{33}$. Ainsi, si les discours politiques se focalisaient sur la sexualité masculine juvénile, dans la pratique, les jeunes femmes furent les premières victimes de la révolution active. Cette mission de purification révolutionnaire fut stoppée dix-huit mois plus tard par un coup d'État militaire qui renversa le régime socialiste.

31 ANM, fonds du Bureau politique national de l'US-RDA BPN, carton 146 : 568 dossier de la Milice populaire 1964-1968, lettre du milicien M.C. du 3e Contingent noyau de Dar-Salam au Camarade Secrétaire permanent de la Milice populaire, Bamako, 24 juin 1968.

32 «Première mesure à prendre dans la lutte contre la crise juvénile », 19 mai 1967, L'Essor, p. 1.

33 Aucun document d'archives ne mentionne de viols commis durant ces événements mais certains témoins masculins interrogés pendant mes recherches les évoquaient à demi-mot, signe du tabou qui entourent aujourd'hui encore les violences sexuelles : "[Les filles] on les maltraitait aussi, on les faisait crier, on violait je crois même... ». Entretien avec Fofana réalisé à Kati, mars 2010. En 1967 il était âgé de 18 ans et était un lycéen actif au sein de l'organisation de jeunesse du Parti à Kayes. Il fut lui-même arrêté par la milice pour avoir porté une tenue « antirévolutionnaire». 


\section{Responsabiliser les filles !}

\section{Éducation sexuelle et contraception dans les années 1970}

L'arrivée des militaires au pouvoir le 19 novembre 1968 fit souffler un vent de liberté paradoxal pour la jeunesse scolarisée: s'il lui était désormais fermement interdit de s'occuper de politique, l'accès au plaisir ne lui était plus défendu. Les militaires - bien plus jeunes que les cadres du régime déchu ${ }^{34}$ - se réclamaient d'une gestion pragmatique et technique des problèmes sociaux ${ }^{35}$. En ce sens, l'encadrement moral et strictement répressif des comportements sexuels juvéniles, qui avait primé dans les années 1960, fut progressivement supplanté par un encadrement sanitaire et éducatif. En 1970, la diffusion du film de Pierre Chevalier, Nathalie. L'Amour s'éveille fut autorisée dans un CinéClub de Bamako ${ }^{36}$. Véritable événement, le film obtint le soutien du journal gouvernemental qui en fit le support d'une campagne d'éducation sexuelle, malgré la polémique générée dans ses colonnes par l'évocation ouverte, et peu moraliste pour l'époque, des premiers rapports sexuels juvéniles ${ }^{37}$. L'année suivante, l'accord fut donné aux Canadiens du Centre de recherche pour le développement international (CRDI) pour la mise en place d'un projet pilote de planning familial à Bamako auquel le régime socialiste s'était préalablement opposé. Cette initiative, émanant de médecins maliens et de l'infirmière Inna Sissoko en charge du Secrétariat aux Affaires sociales dans le gouvernement militaire de Moussa Traoré, obligea à abroger les articles de loi hérités

34 Les militaires qui prirent le pouvoir en 1968 étaient âgés d'une trentaine d'années.

35 Rillon 2015.

36 Pierre Chevalier, Nathalie. L'amour s'éveille, 1969, 88'. "Premier film français d'éducation sexuelle » selon son affiche publicitaire. Ce film, réalisé en 1968, avait été interdit de sortie dans un premier temps. Il fut soutenu par le Mouvement français pour le planning familial et sa diffusion fut finalement autorisée avec cependant la censure de certaines scènes et son interdiction aux moins de 18 ans. Il fut diffusé au Mali par le Ciné-Club Askia Nouh qui venait d'être créé par des militants d'une organisation clandestine.

37 Diabate Massa Makan, «L'éducation sexuelle : patience, méthode, exigence », L'Essor, 11 mars 1970, p. 1 et p. 4 ; Comité directeur du Ciné-Club Askia Nouh, "Un film qu'il faut aborder sans préjugés, avec un esprit mûr et critique », L'Essor, 12 mars 1970, p. 1 et p. 4.; Doucoure B.B., «Nécessité de l'information et de l'éducation sexuelles (sic)», L'Essor, 19 mars 1970, p. 1 et p. 4. 
de l'époque coloniale qui interdisaient la contraception ${ }^{38}$. Le Mali devint ainsi le premier pays d'Afrique subsaharienne francophone à initier une telle politique, étendue de Bamako aux capitales régionales en 1973. La même année, le ministère de l'Éducation nationale organisait le premier séminaire interafricain sur l'éducation sexuelle. Sous couvert de protection maternelle et infantile, c'est la sexualité des jeunes - et plus particulièrement des scolaires - qui était au cœur des débats. Loin de se limiter à stigmatiser les comportements juvéniles, les responsables politiques, les éducateurs et le personnel sanitaire plaidaient désormais en faveur de la mise en place de cours d'éducation sexuelle dès l'école primaire, de l'instauration de la mixité dans les classes qui «contribuerait avantageusement à rapprocher garçons et filles, donc à estomper ce recul du jeune par rapport à son corps $»^{39}$, et allaient même jusqu'à envisager l'accès des jeunes (garçons et filles) à la contraception. La militante voltaïque Jacqueline Ki-Zerbo, dont l'exposé fut le plus applaudi, défendait l'idée que « la contraception est un facteur de libération de la femme $»^{40}$. Face aux garçons qui pensaient que « la grossesse c'est le brevet de la virilité $»^{41}$, il paraissait déraisonnable de demander aux jeunes filles de faire vœu d'abstinence en dehors du mariage ; mieux valait leur donner les connaissances et les moyens de disposer de leur corps afin qu'elles puissent poursuivre leurs études, comme le notait un rapport de 1975 :

Les règlements hérités du régime français exigent qu'une élève du secondaire enceinte quitte l'école, sans espoir de la réintégrer plus tard au même niveau. Chaque année, une proportion non négligeable d'étudiantes au niveau du baccalauréat doit abandonner l'école et renoncer aux avantages acquis par des années d'effort. Les dilemmes moraux et politiques posés par cette situation doivent encore être résolus, mais il ne fait aucun doute que les catastrophes qu'elle produit ont contribué à intensifier la demande pour la planification familiale ${ }^{42}$.

\footnotetext{
38 Van de Walle \& Maïga 1991.

39 CRDI 1973: 90. Il s'agit du compte-rendu du séminaire interafricain sur l'éducation sexuelle tenu à Bamako du 16 au 25 avril 1973.

40 CRDI $1973: 91$.

41 CRDI $1973: 89$ et 95 .

42 «Regulations inherited from the French regime require that a secondary school student who becomes pregnant leave school, with no hope of re-entering later at
} 
Si ces discours témoignaient d'une révolution des cadres de pensée des adultes, la mise en application d'une politique d'accès à la contraception pour les jeunes filles se heurtait à de nombreuses difficultés. L'installation d'une clinique en plein centre de Bamako, délivrant gratuitement des contraceptifs (stérilets, pilules et préservatifs), avait suscité la colère d'une partie de la population bamakoise et des responsables religieux en particulier. Le personnel du planning était accusé de « faire des avortements [...] La population était là à dire qu'on prostituait leurs femmes » raconte sa première secrétaire $^{43}$. Pour l'une des sages-femmes de la structure, "il fallait être fou pour avoir une telle initiative dans un pays aussi musulman que le Mali ${ }^{44}$, évoquant par ces mots le profil atypique de son initiateur Faran Samaké, directeur de l'hôpital psychiatrique de Bamako et grand ami du responsable de la sécurité Tiékoro Bagayoko, dont les services furent mis à profit pour intimider les opposants au planning. Afin de ne pas exacerber les tensions, l'accès au planning familial de Bamako fut, à ses débuts, exclusivement réservé aux célibataires majeures ou aux femmes mariées, ces dernières devant justifier d'une autorisation maritale :

Pour se faire accepter au planning familial au début, il fallait nécessairement que ton mari t'accompagne ou bien te donne sa carte d'identité accompagnée d'une procuration comme quoi il est d'accord pour que sa femme soit planifiée $[s i c]$. Ce document était épinglé au dossier de la cliente pour qu'un jour, si le mari venait à se plaindre... ${ }^{45}$.

the same level. Every year, an appreciable proportion of students at the baccalauréat level have to drop out of school and give up the advantages acquired through years of effort. The moral and political dilemmas posed by this situation have yet to be resolved, but there is no doubt that the catastrophe it produces have helped to intensify the demand for family planning ». Laplante, Samaké \& Brown 1975 : 10 (traduction personnelle).

43 Entretien avec Oumou Keita secrétaire du Planning familial de 1972 à 2007, Bamako, ${ }^{\text {er }}$ octobre 2014.

44 Entretien avec Marcelle Richard, sage-femme au Planning familial de 1972 au début des années 1990, métisse de confession catholique. Bamako, 3 octobre 2014.

45 Entretien avec Oumou Keita, secrétaire du Planning familial de 1972 à 2007, Bamako, $1^{\text {er }}$ octobre 2014. 
Mais, contrairement à ce que pourrait laisser entendre ce témoignage, les femmes mariées n'étaient pas les seules à fréquenter le planning. Les dossiers personnels conservés dans les archives de l'association montrent que les jeunes femmes célibataires de moins de 25 ans représentaient plus du tiers des patientes dans les années 197046. Parmi elles, les scolaires arrivaient en tête (53\%). Le profil des patientes était le reflet de l'évolution des pratiques matrimoniales en milieu urbain allant dans le sens d'une accentuation du célibat, singulièrement chez les femmes âgées de moins de $25 \mathrm{ans}^{47}$. Donner accès à la contraception à ces jeunes célibataires était envisagé par les nouvelles autorités comme un enjeu social et sanitaire visant à limiter les infanticides et les abandons d'enfants. À la lecture des archives de police et de gendarmerie, rien n'indique pourtant que ces pratiques aient augmenté dans les années $1970^{48}$. C'était cependant l'argument dont avait usé Inna Sissoko pour convaincre ses collègues masculins de l'urgence de mettre en place une politique d'accès à la contraception ${ }^{49}$. C'était aussi en ce sens qu'une sociologue malienne, dont la thèse fut publiée par la maison d'édition gouvernementale en 1978, plaidait en faveur " d'une politique sociale de contraception » et appelait les responsables politiques à faire changer les mentalités à ce sujet, car « il est illusoire de vouloir supprimer l'activité sexuelle chez [les jeunes filles] avec succès au point où elles en sont $\aleph^{50}$. Les jeunes

46 Archives de l'Association malienne pour la protection et la promotion de la famille (AMPPF). Ont été consultés les dossiers personnels des patient.e.s venu.e.s à la clinique en 1972, 1973 et 1979. Soit un total de 179 femmes et 8 hommes.

47 Entre 1960 et 1976, l'âge moyen des femmes au premier mariage passe de 16,6 ans à 19,7 ans et la proportion de célibataires parmi les 20-24 ans passe de $4 \%$ à $24 \%$. Du côté des hommes, l'âge au premier mariage est beaucoup plus élevé, passant de 27,4 ans à 29,2 ans. Marcoux, Gueye \& Konaté 1995 : 123.

48 Les affaires d'infanticides jugés au Tribunal de Bamako entre 1967 et 1974 varient de zéro à un cas par an ; et on ne compte qu'un seul cas entre 1970 et 1974. Sangaré 1978 : 50 . Aucun cas n'est signalé dans les archives du ministère de l'Intérieur.

49 «Parce qu'au moment où Inna avait demandé ça, il y avait trop d'abandon d'enfants, d'infanticides... [L'orphelinat de Bamako] la Pouponnière était pleine et c'est les Affaires sociales qui s'occupaient de la Pouponnière. [...] C'est cette raison qui l'a poussée à mettre en place le planning familial », entretien avec Oumou Keita, secrétaire du Planning familial de 1972 à 2007, Bamako, 1er octobre 2014.

50 Sangaré $1978: 52$. 
hommes étaient les grands absents de cette campagne de prévention des grossesses hors mariage, où la hantise de la prostitution féminine avait cédé le pas à celle des « filles-mères ».

Si les jeunes filles des établissements scolaires étaient les premières cibles de cette politique, une analyse plus fine des dossiers du planning témoigne du décalage existant entre les discours et la pratique. Sur les 112 patientes ayant fréquenté la clinique entre 1972 et 1973 , on ne compte que onze filles scolarisées (soit 10\%), soit une lycéenne âgée de 20 ans et dix élèves du cycle fondamental, âgées de 17 à 23 ans, «fillesmères » pour sept d'entre elles. La présence d'une mineure ${ }^{51}$ indique que les entorses au règlement demeuraient possibles, à condition que les élèves soient informées de l'existence d'une telle structure. Mais, afin de ne pas embraser les esprits, le personnel avait fait le choix de ne pas ébruiter l'existence du planning : ni sensibilisation dans les écoles et les quartiers ni publicisation par le journal et la radio gouvernementale n’étaient envisagées. Seul «le bouche-à-oreille » avait attiré ces femmes et huit hommes mariés à la clinique. Six années plus tard, la proportion de jeunes filles s'était singulièrement accrue parmi les patientes du planning. Sur les 65 femmes reçues en 1979, 24 étaient scolarisées et plus de la moitié étaient des lycéennes. Un père, gendarme, y avait même envoyé ses trois filles scolarisées et âgées de 15 ans à 18 ans pour qu'elles se fassent prescrire une contraception. Bien que les mineures demeurent minoritaires, leur taux de fréquentation s'était aussi développé avec neuf patientes âgées de 15 ans à 17 ans. À l'inverse, on ne trouve plus aucun dossier d'hommes ayant fréquenté le planning.

La proportion croissante de scolaires à venir consulter le planning était autant le signe que «le bouche-à-oreille» fonctionnait, que le fruit d'une mobilisation spécifique de ces jeunes filles qui revendiquèrent, à partir de 1978, «l'arrêt de l'exclusion des fillesmères des établissements scolaires ». Deux décennies après l'indépendance du pays, cette réglementation héritée des écoles coloniales était ouvertement contestée. Cette prise de parole publique et collective de la part d'individus qui cumulaient un double statut de mineures - jeunes et femmes à la fois - constituait un véritable

51 Suivant l'âge de la majorité matrimoniale (pour les filles) et de la majorité pénale fixées à 18 ans. La majorité civile était cependant fixée à 21 ans pour les deux sexes. 
tournant. Cette revendication avait émergé dans le contexte d'un vaste mouvement social des élèves et étudiant.e.s initié en 1977 contre la réforme du système éducatif et qui fit vaciller, pour la première fois, le régime militaire. Comment cette revendication a-telle émergé au sein de l'organisation ? Par qui fut-elle portée ? Fut-elle débattue ? Malheureusement, les données manquent pour répondre à ces interrogations. Néanmoins, ce ne fut certainement pas un hasard qu'une revendication féminine ait été inscrite sur la plateforme étudiante au moment même où Bintou Maïga, dite "Winnie Mandela » accédait au bureau de coordination de l'organisation étudiante, instance exclusivement masculine jusque-là. Interpellé sur cette question à de multiples reprises par les élèves, le gouvernement se vit obligé de prendre publiquement position. Dans une interview accordée à L'Essor, le lieutenant-colonel Yousouf Traoré, alors ministre de l'Éducation nationale affirmait qu'il s'agissait d'un phénomène minoritaire. Tout en reconnaissant qu'il s'agissait d'une question «très sérieuse et délicate qui mérite une attention particulière $»^{52}$, le ministre balayait la revendication de la réintégration des jeunes filles concernées en promouvant la «prévention» et l'introduction de l'éducation sexuelle dans les programmes scolaires «afin que les jeunes filles soient mieux informées». À ses yeux, les garçons n'étaient pas concernés : ce problème était lié à l'ignorance, voire au libertinage, des jeunes filles. Cette vision de la sexualité, qui considérait les filles comme seules responsables de la procréation, se situait aux antipodes de celle de l'organisation étudiante. Celle-ci pointait du doigt l'inégalité de traitement dont étaient « victimes » les jeunes filles enceintes et réclamait «que soient cherchés des moyens adéquats en vue de trouver une solution à ce fléau social dont la plupart de nos sœurs sont victimes alors que les mauvais garçons qui sont coupables demeurent impunis $»^{53}$. Étonnamment, l'organisation

52 «L'éducation au Mali. Le campus, les filles-mères, l'UNEEM et les mouvements estudiantins. Une interview du Lt-colonel Youssouf Traoré », L'Essor, 13-14 mai 1978, p. 4.

53 «Résolution finale de la réunion de l'UNEEM. Adhésion aux idéaux de l'UNJM mais autonomie pour les problèmes scolaires ", L'Essor, 28-29 janvier 1978, p. 4. Cette asymétrie face à la grossesse des écolières était aussi dénoncée 
étudiante demeurait silencieuse sur la question contraceptive ${ }^{54}$. Promouvant une sorte d'idéal familialiste déconnecté du cadre légal du mariage, elle appelait les élèves et les étudiants masculins à assumer leurs responsabilités paternelles. L'association maintint la revendication dans la plate-forme défendue par les étudiant-e-s au cours de l'été 1979, en arguant que la maternité ne devait pas constituer un frein supplémentaire à la scolarisation des jeunes filles. Quelques mois plus tard, l'ensemble du mouvement étudiant fut brutalement réprimé, en même temps que le gouvernement décidait d'introduire l'éducation sexuelle dans les lycées et de lever l'autorisation maritale pour les patientes du planning. Les aînées mariées, qui ne s'étaient pas mobilisées, gagnaient en autonomie, tandis que la sexualité des lycéennes était placée sous le contrôle des éducatrices et que celle des cadettes scolaires, les écolières, était occultée.

Dans les années 1980 cependant, la vie sexuelle des jeunes filles scolarisées est devenue un fait socialement admis. L'enjeu pour les adultes était désormais de limiter les comportements à risques et, ainsi, de prévenir les grossesses précoces des élèves. C'est dans ce sens que fut créé en 1987 un magazine à destination des jeunes, accordant une place de premier plan à l'éducation sexuelle (contraception féminine et masculine, explication des organes génitaux et du système de reproduction, dessins à l'appui). Selon les initiateurs de ce journal : "Beaucoup des jeunes des deux sexes forment des couples et "vivent ensemble", parfois pendant longtemps. Ces jeunes ont souvent des

individuellement par les jeunes filles concernées dans le Kenya des années 1960. Thomas 2006b.

54 Peut-être était-ce une manière de se démarquer des adultes qui avaient porté la revendication et, en particulier, de l'organisation des femmes proche du pouvoir? On peut aussi interpréter ce silence comme le signe avant-coureur d'une critique, qui va devenir de plus en plus audible dans les années 1980 parmi les jeunes militant-e-s marxistes et anti-impérialistes, à l'encontre des effets secondaires des pilules contraceptives distribuées en Afrique. Certain.e.s voyaient aussi derrière la politique de planification une forme d'eugénisme occidental «afin que les pauvres ne fassent plus d'enfants». Entretien avec Rokia Sanogo, militante politique et professeure à l'Université de pharmacie, Bamako, le 9 février 2006. 
rapports sexuels qu'ils conditionnent rarement à des règles $»^{55}$. Si l'encadrement de la sexualité juvénile demeurait une préoccupation constante, la norme de l'abstinence énoncée jusqu'à la fin des années 1960 avait cédé le pas à une acceptation de l'initiation avant le mariage, même pour les filles, et à la diffusion d'une éducation sexuelle semblable pour les deux sexes.

Tout au long des années 1960-1970, la question de la sexualité juvénile en milieu scolaire n'a cessé d'interpeller et d'inquiéter les autorités politiques. Le poids accordé à ce sujet était intimement lié à la place dévolue à la jeunesse scolarisée, à son encadrement et à sa formation tant morale qu'intellectuelle, dans le cadre de la construction nationale malienne. C'est ainsi que les dirigeants de la Ire République socialiste, qui assignaient un rôle politique primordial à cette catégorie sociale, ont développé une gestion exclusivement répressive de sa sexualité alors assimilée à une déviance dangereuse pour la stabilité de l'ordre social. En ce sens, la moralisation sexuelle des jeunes hommes, qui intervint dans le contexte de la révolution culturelle malienne, témoigne du lien profond tissé par les autorités entre la régénération de la Nation et celle de ses futures élites masculines. Mais cet article a aussi montré que les jeunes n'avaient pas été totalement absents des débats qui entouraient leur sexualité. Les filles scolarisées en particulier, dont l'initiation à la sexualité suscitait les plus vives condamnations, ont mené des combats feutrés pour conquérir un droit au plaisir. Initiée à l'époque coloniale tardive, l'aspiration à une autonomie sexuelle s'est faite de plus en plus audible au fil du temps, et participa à alimenter les craintes des adultes. Paradoxalement, cette peur a aussi obligé les pouvoirs publics à prendre des mesures pour prévenir les grossesses des élèves du secondaire et à se départir d'une vision moraliste qui avait primé sous le régime socialiste. Avec le changement de régime en 1968, la gestion technique des problèmes sociaux a pris le pas sur la morale sexuelle et favorisé la mise en place d'une politique d'éducation sexuelle et de

55 «L'éducation sexuelle. Introduction à la contraception», Grin-Grin. Le magazine des jeunes, n², février 1987, p. 16. 
légalisation de la contraception. La focale était désormais mise sur les filles, dont le droit à la sexualité était ainsi reconnu à condition qu'elles sachent discipliner et maitriser leur fécondité. Au Mali, comme ailleurs ${ }^{56}$, ces deux décennies ont donc été marquées par une évolution des dispositifs de contrôle de la sexualité juvénile, faisant reposer sur les filles - plutôt que sur les garçons - l'intériorisation des normes et des recommandations en matière de comportements sexuels.

\section{Bibliographie}

AlLman Jean, 1996, « Rounding up spinsters: gender chaos and unmarried women in colonial asante ", The Journal of African History, 37/2, p. 195-214.

BÂ Mariama, 2007, Une si longue lettre, Dijon, Le Rocher.

BARTHÉLÉMY Pascale, 2004, «Femmes, africaines et diplômées : une élite auxiliaire à l'époque coloniale. Sages-femmes et institutrices en Afrique occidentale française (1918-1957) », thèse d'histoire, Université Paris 7 [publiée, en 2010, aux Presses universitaires de Rennes].

Bozon Michel, 2012, «Autonomie sexuelle des jeunes et panique morale des adultes. Le garçon sans frein et la fille responsable », Agora débats/jeunesses, 1/60, p. 121-134.

—, 2013, Sociologie de la sexualité, Paris, Armand Colin.

Centre de recherche pour le développement international (CRDI), 1973, Éducation sexuelle en Afrique Tropicale, Ottawa, CRDI.

Diouf Mamadou, 2003, «Engaging postcolonial cultures: African youth and public space », African Studies Review, 46/2, p. 1-12.

GarY-TounKara Daouda, 2003, «Quand les migrants demandent la route, Modibo Keita rétorque: "Retournez à la terre !". Les Baragnini et la désertion du "Chantier National" (1958-1968) ", Mande Studies, 5, p. 49-64.

Goerg Odile, 2007, «Femmes adultères, hommes voleurs ? La "justice indigène" en Guinée », Cabiers d'études africaines, 3/187-188, p. 495-522.

JeAn-BAPTISTE Rachel, 2014, Conjugal Rights: marriage, sexuality, and urban life in Colonial Libreville, Gabon, Athens, Ohio University Press.

Laplante André, Samaké Faran \& George F. Brown, 1975, Family Planning in Mali, Ottawa, International Development Research Center.

56 Bozon 2013 : 35-36. 
Marcoux Richard, Gueye Mouhamadou \& Mamadou Kani KonAté, 1995, «La nuptialité: entrée en union et type de célébration à Bamako», in Dieudonné Ouedraogo \& Victor Piche (dir.), L'Insertion urbaine à Bamako, Paris, Karthala, p. $117-144$.

MORIN Edgar, 1969, "Culture adolescente et révolte étudiante », Annales. Économies, Sociétés et Civilisations, 24/3, p. 765-776.

REBREYEND Anne-Claire, 2010, "France. Les "Filles amoureuses" : une nouvelle catégorie des années 1950 », in Véronique Blanchard, Régis ReVEnIN \& JeanJacques Yvorel (dir.), Les Jeunes et la sexualité. Initiations, interdits, identités, Paris, Éditions Autrement, p. 300-310.

République du Mali, 1996, Enquête Démographique et de Santé Mali 1995-1996, Calverton, Maryland USA.

République du Mali, 2007, Enquête Démographique et de Santé Mali 2006, Calverton, Maryland USA.

REVENIN Régis, 2015, Une Histoire des garcons et des filles. Amour, genre et sexualité dans la France d'après-guerre, Paris, Vendémiaire.

RILLON Ophélie, 2010, «Corps rebelles : la mode des jeunes urbains dans les années 1960-1970 au Mali », Genèses, 4/81, p. 64-83.

— 2015, «Mobilisations féminines en contexte autoritaire. La "dépolitisation” comme outil d'émancipation dans le Mali des années 1970 », in Michel CAHEN, MarieEmmanuelle Pommerolle \& Emmanuelle Kadya Tall (dir.), Mobilisations collectives en Afrique : contestations, résistances, révolutions et révoltes, Leiden, Brill, p. 164-182.

RodeT Marie \& Brandon CounTy, 2016, «Genre, génération et contrôle de la circulation des personnes entre le Mali et le Sénégal à l'heure de Modibo Keita (1959-1968) ", in Muriel Gomez-Perez (dir.), Femmes, génération et agency en Afrique subsaharienne : vers de nouveaux défis, Paris, Karthala (à paraître).

SANGARÉ Aminata Sanankoua, 1978, La Délinquance juvénile au Mali, Bamako, Éditions Populaires [issue d'une thèse soutenue à l'Université Paris V en 1976].

SoHn Anne-Marie, 2001, Agge tendre et tête de bois : histoire des jeunes des années 1960, Paris, Hachette.

THOMAS Lynn, 2006a, «Gendered reproduction: placing schoolgirl pregnancies in african history », in Stephen Miescher, Catherine Cole \& Takyiwaa ManuH (eds.), Africa after Gender?, Bloomington, Indiana University Press, p. 48-62.

—, 2006b, «School girl pregnancies, letter-writing, and 'modern' persons in Late Colonial East Africa ", in Karin BArBer (ed.), Africa's Hidden Histories: everyday literacy and making the self, Bloomington, Indiana University Press, p. 180-207.

VAN DE WaLle Francine \& Mariam MaÏGA, 1991, «Family planning in Bamako, Mali », International Family Planning Perspectives, 17/3, p. 84-90. 
